\title{
Myasthenia gravis: a pupillometric study
} Dimitrios Tsiptsios*, Dimitrios Fotiou, Maria Nakou, Vasilios Stergiou, Aggelis Fotiou, Irene Kalliolia, Evangelia Giza, Evangelia Theodoridou, Catherine Brozou and Charalambos Giantselidis

\author{
Address: Laboratory of Clinical Neurophysiology, Aristotle University of Thessaloniki, Greece \\ * Corresponding author
}

\author{
from International Society on Brain and Behaviour: 2nd International Congress on Brain and Behaviour \\ Thessaloniki, Greece. 17-20 November 2005 \\ Published: 28 February 2006 \\ Annals of General Psychiatry 2006, 5(SuppI I):S320 doi:I0.I 186/I744-859X-5-SI-S320
}

\section{Background \\ Myasthenia gravis (MG) is a disease of the neuromuscular junction (NMJ) that, in its typical form, is caused by autoimmune on the postsynaptic receptors of Acetylcho- line (AChRs) of the Peripheral Nervous System (PNS)1. We investigate the effect of MG on the Central Nervous System (CNS) and the pupillary smooth muscle.}

\section{Materials and methods}

40 Myasthenic patients, recently diagnosed with MG based on their clinical features, electrodiagnostic tests and high titers of anti- AChRs took part in this research and 40 healthy subjects of matching age and gender underwent a pupillometric study in both eyes, first to single flash stimuli of 24.6 candelas/m2 intensity and $20 \mathrm{msec}$ duration and secondly to multiple repetitive flash stimuli of the same intensity and duration whose frequency was increased every 30 seconds from 0.6 to $1.8 \mathrm{~Hz}$ with successive steps of $0.3 \mathrm{~Hz}$.

The pupillometric parameters that were studied are: 1 . Initial Radius, 2. Latent Period, 3. Time to Maximum Contraction, 4. Amplitude of the Contraction, 5. Maximum Speed and Maximum Acceleration of the Contraction.

\section{Results}

A statistically significant increase in the Latent Period and a decrease in the Amplitude, the Maximum Speed and Maximum Acceleration to single flash stimuli and a decrease in the Amplitude of the Pupil's Oscillations to multiple repetitive flash stimuli was observed in Myasthenic patients, in contrast to the same parameters in the non- Myasthenic subjects.

\section{Discussion}

It is widely believed that MG is a disease of the PNS and the striated muscles, so that the CNS and the smooth muscles are not affected. On the other hand, many previous studies propose a dysfunction of the AChRs not only in the PNS, but also a disorder of the AChRs that are located in the CNS.

The Myasthenic Pupils are characterized by a "sluggish" reaction, as indicated by the increase in the Latent Period and the decrease in the Amplitude, the Maximum Speed and the Maximum Acceleration to single flash stimuli and the Amplitude of the Pupil's Oscillations to multiple repetitive flash stimuli. These findings may reflect a reduction in the amount of Acetylcholine that is available in the NMJ of the pupil's smooth muscle or a broader Central Cholinergic deficit2. This hypothesis is reinforced by the restoration of the predictable values of the parameters under study after the administration of anti-acetylcholinesterase drugs.

\section{References}

I. Fotiou F: Practical use of electrophysiological and optical methods in evaluating neurological, neuro-opthalmological and neuro-psychiatric diseases Edited by: Parisianos G. Athens; 1999.

2. Fotiou F, Fountoulakis KN: Evidence for a central cholinergic deficit in myasthenia gravis. J Neuropsychiatry Clin Neurosci 2000, | 2:5|4-5|5. 